\title{
Rapid growth and osteogenic differentiation of mesenchymal stem cells isolated from human bone marrow
}

\author{
WAN NAZATUL SHIMA ${ }^{1,2}$, ABDUL MANAF ALI ${ }^{3}$, TAMILSELVAN SUBRAMANI $^{1}$, \\ NOORJAHAN BANU MOHAMED ALITHEEN ${ }^{1}$, MUHAJIR HAMID ${ }^{4}$, \\ ABDUL RANI SAMSUDIN $^{2}$ and SWEE KEONG YEAP

\begin{abstract}
${ }^{1}$ Department of Cell and Molecular Biology, Faculty of Biotechnology and Biomolecular Sciences, Universiti Putra Malaysia, Serdang, Selangor 43400; ${ }^{2}$ School of Dental Sciences, Universiti Sains Malaysia Health Campus, Kubang Kerian,

Kelantan 16150; ${ }^{3}$ Faculty of Agriculture and Biotechnology, Universiti Zainal Abidin, Kampus Kota,

Kuala Terengganu, Terengganu 20400; ${ }^{4}$ Department of Microbiology, Faculty of Biotechnology and Biomolecular Sciences;

${ }^{5}$ Institute of Bioscience, Universiti Putra Malaysia, Serdang, Selangor 43400, Malaysia
\end{abstract}

Received March 10, 2014; Accepted September 11, 2014

DOI: $10.3892 / \mathrm{etm} .2015 .2381$

\begin{abstract}
Mesenchymal stem cells (MSCs) are involved in bone formation in the embryo, bone repair and remodeling. The differentiation of these cells is a complex multistep pathway that involves discrete cellular transitions and is similar to that which occurs during hematopoiesis. MSCs have self-renewal capacity without differentiation in long-term culture. In the present study, MSCs were isolated from human bone marrow and characterized by the presence of cluster of differentiation 105 marker using the labeled streptavidin biotin method. The MSCs were cultured in Dulbecco's modified Eagle's medium supplemented with fetal bovine serum, ascorbic acid, $\beta$-glycerol phosphate and dexamethasone to differentiate into osteoblasts. Biological in vitro analysis showed the rapid proliferation of the MSCs. Further evaluation of specific osteogenic markers using von Kossa staining and the alkaline phosphate assay demonstrated that the MSCs were stimulated to differentiate into osteoblast-lineage cells. This mesengenic potential indicated that the bone marrow-derived cells were multipotent MSCs. The findings of this study show that bone marrow can be a legitimate source of MSCs for the production of osteoblasts for utilization in bone replacement therapy.
\end{abstract}

Correspondence to: Dr Noorjahan Banu Mohamed Alitheen, Department of Cell and Molecular Biology, Faculty of Biotechnology and Biomolecular Sciences, Universiti Putra Malaysia, Serdang, Selangor 43400, Malaysia

E-mail: noorjahan@biotech.upm.edu.my

Key words: bone marrow, cell differentiation, mesenchymal stem cell, osteoblast

\section{Introduction}

The formation of bone in the embryo and during adult fracture repair and remodeling involves the progeny of cells known as mesenchymal stem cells (MSCs). These cells continuously undergo self-replication, while a number become committed to mesenchymal cell lineages, such as bone, cartilage, tendon, ligament and muscle. The differentiation of these cells within each of the mesenchymal cell lineages is a complex multistep pathway that involves discrete cellular transitions and is similar to that which occurs during hematopoiesis. The progression of the cells between stages depends on the presence of specific bioactive factors, nutrients and other environmental cues, which coordinate to elicit the entire differentiation process (1). MSCs have self-renewal capacity without differentiation in long-term culture. Under certain conditions, MSCs can differentiate into adipocytes, chondrocytes, astrocytes, tenocytes, cardiomyocytes, hepatocytes, neurons and muscle, endothelial and endodermal cells (2) MSCs have generated considerable attention and shown promise as a potential source of cells for cell-based therapeutic strategies, primarily owing to their intrinsic ability to self-renew and differentiate into functional cell types that constitute the tissue in which they exist. MSCs are considered a readily accepted source of stem cells, since such cells have already demonstrated efficacy in multiple types of cellular therapeutic strategies, including applications in treating children with osteogenesis imperfecta (3), hematopoietic recovery (4) and tissue regeneration strategies (5). An osteoblast is a bone-forming cell that arises from MSCs. These cells are known to exist in the periosteum and bone marrow, but their population is extremely small. Culture-expanded MSCs have the in vitro capacity to differentiate into osteoblasts and, notably, the cultured osteoblast can form extracellular matrix in culture (6).

Despite the significant interest in MSCs, there remains no established protocol for the isolation and expansion of the cells in culture. In the majority of experiments, isolated MSCs from bone marrow mononuclear cells (MNCs) were determined 
Table I. Serial dilution of $p$-nitrophenol standard $(200 \mu \mathrm{g} / \mathrm{ml})$ for concentrations of 0-200 $\mu \mathrm{g} / \mathrm{ml}$.

\begin{tabular}{lccr}
\hline $\begin{array}{l}\text { Serial dilution standard } \\
p \text {-nitrophenol }\end{array}$ & $\begin{array}{c}\text { Volume of } 200 \mu \mathrm{g} / \mathrm{ml} \\
\text { solution }(\mu \mathrm{l})\end{array}$ & $\begin{array}{c}\text { Volume of distilled } \\
\text { water }(\mu \mathrm{l})\end{array}$ & $\begin{array}{c}\text { Concentration } \\
(\mu \mathrm{g} / \mathrm{ml})\end{array}$ \\
\hline A & 0.000 & 200.0 & 0.000 \\
B & 3.125 & 196.9 & 3.125 \\
C & 6.250 & 193.8 & 6.250 \\
D & 12.500 & 187.5 & 12.500 \\
E & 25.000 & 175.0 & 25.000 \\
F & 50.000 & 150.0 & 50.000 \\
G & 100.000 & 100.0 & 100.000 \\
H & 200.000 & 0.0 & 200.000 \\
\hline
\end{tabular}

based on their tight adherence to tissue culture plastic $(7,8)$. These isolated cells were initially heterogeneous, and difficult to distinguish from other adherent cells. None of the several methods that have been developed to prepare more homogenous populations (7) have thus far earned wide acceptance. In the present study we aimed to slightly modify the method by Friedenstein et al (8) and successfully differentiate MSCs into bone cells. Several types of materials, including allograft, xenograft and synthetic biomaterials, are currently used for bone replacement therapy, but the future of bone reconstruction lies in the use of stem cells for bone development. The aim of the present study was to analyze the rapid growth and osteogenic differentiation of MSCs isolated from human bone marrow.

\section{Materials and methods}

Bone marrow MSC isolation and primary culture. Human bone marrow was obtained with the informed consent of patients at the Hospital Universiti Sains Malaysia (Kubang Kerian, Malaysia). The marrow material was aspirated from the iliac crest and collected in a 50-ml centrifuge tube containing citrate phosphate buffer ( $\mathrm{pH} 7$; Sigma-Aldrich, St. Louis, MO, USA) with anticoagulant and processed within $15 \mathrm{~h}$ of collection. The bone marrow was flushed with the primary culture media, which contained Dulbecco's Modified Eagle's Medium (DMEM; Gibco-BRL, Grand Island, NY, USA), $10 \%$ fetal bovine serum (FBS; HyClone, Logan, UT, USA) and $2 \%$ antibiotics $(100 \mathrm{U} / \mathrm{ml}$ penicillin and $100 \mathrm{U} / \mathrm{ml}$ streptomycin). Nucleated cells were collected by density gradient centrifugation onto a Percoll solution (density, $1.073 \mathrm{~g} / \mathrm{ml}$; Sigma-Aldrich). The cells were seeded into a $50-\mathrm{ml}$ cell culture flask and incubated at $37^{\circ} \mathrm{C}$ in $5 \% \mathrm{CO}_{2}$. The media was first replaced after $48 \mathrm{~h}$ when the cells reached confluence and subsequently twice a week. Continuous passage was performed in order to harvest relatively pure MSCs. The present study was approved by the Institutional Ethical Committee of the Universiti Putra Malaysia (Serdang, Malaysia).

Characterization of bone marrow MSCs. The third-passage MSCs were characterized with respect to the expression of surface antigens. The cells were seeded at $3.1 \times 10^{3} / \mathrm{ml}$ in two-well chamber slides in $1 \mathrm{ml}$ MSC growth medium until cells became confluent. The cells grown on slides were fixed with $4 \%$ formaldehyde for $30 \mathrm{~min}$ and then incubated in $1 \mathrm{ml}$ 1:5 diluted mouse anti-human cluster of differentiation 105 (CD 105; DakoCytomation, Glostrup, Denmark). The cells were subsequently rinsed gently with Tris buffered saline (TBS) and replaced in fresh TBS. Sufficient link solution (biotinylated anti-rabbit, anti-mouse and anti-goat immunoglobulins in phosphate-buffered saline containing stabilizing protein and $0.015 \mathrm{~mol} / 1$ sodium azide; DakoCytomation, Glostrup, Denmark) was applied to cover the cells, which were incubated for $15 \mathrm{~min}$. The cells were rinsed with TBS and streptavidin-alkaline phosphatase (ALP) solution was applied to cover the cells, which were incubated for a further $10 \mathrm{~min}$. Following this time period, the cells were counterstained with hematoxylin for $1 \mathrm{~min}$ and observed under the microscope (Eclipse Ti, Nikon, Melville, NY, USA). The positive MSC lines were obtained from the American Type Culture Collection (Rockville, MD, USA) and used as a positive control. For the negative control, the MSCs were not incubated with CD 105.

Cell seeding and culture in vitro. MSC cultures were seeded into a culture plate at a density of $3.1 \times 10^{3} / \mathrm{ml}$. Subsequent to adding the cell suspension with osteogenic differentiation media (DMEM supplemented with $10 \%$ FBS, $10 \mathrm{~mm}$ $\beta$-glycerol phosphate, $0.1 \mu \mathrm{M}$ dexamethasone and $100 \mu \mathrm{M}$ ascorbate-2-phosphate) to the cell culture plate, the plates were incubated for $4 \mathrm{~h}$ for cell attachment. The media was removed and the adherent cells were cultured in fresh osteogenic medium at $37^{\circ} \mathrm{C}$ in $5 \% \mathrm{CO}_{2}$ for three weeks in vitro. The culture media were replaced twice a week.

\section{Osteogenic differentiation of MSCs}

ALP assay. ALP is a vital marker enzyme of the early stages of osteogenic differentiation. After one week of osteogenic differentiation culture, the plate was placed at $-70^{\circ} \mathrm{C}$ for $20 \mathrm{~min}$ and then was transferred to a $37^{\circ} \mathrm{C}$ room for $15 \mathrm{~min}$ to lyse the cell membranes. Serial dilution of $p$-nitrophenol standard was prepared at the concentration of $3.125 \mu \mathrm{g} / \mathrm{ml}$ (Table I) and a volume of $100 \mathrm{ml}$ was distributed into the appropriate wells. A total of $50 \mu \mathrm{l}$ substrate solution was added to all sample wells, and the plate was covered and mixed well for 2 min on the Titertek Plate Shaker (Titertek-Berthold, Pforzheim, 

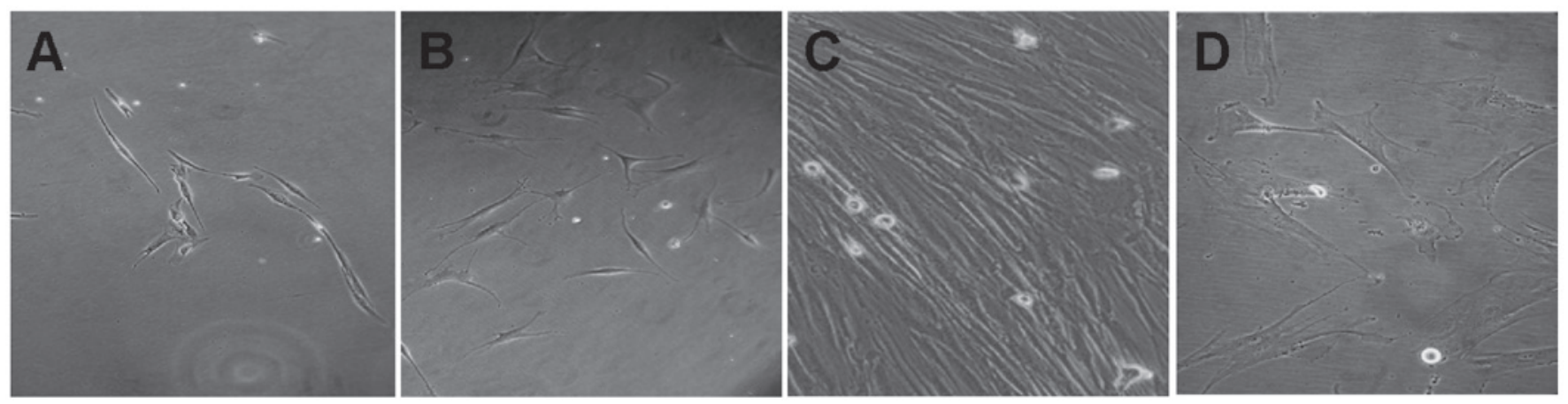

Figure 1. Morphology of MSCs. (A) Early morphology of MSCs following overnight culture (magnification, x100). (B) MSCs after five days of culture (magnification, x100). (C) Confluent MSCs (magnification, x200). (D) Appearance and growth of MSCs in Dulbecco's modified Eagle's medium-10\% fetal bovine serum (magnification, x200). MSC, mesenchymal stem cell.
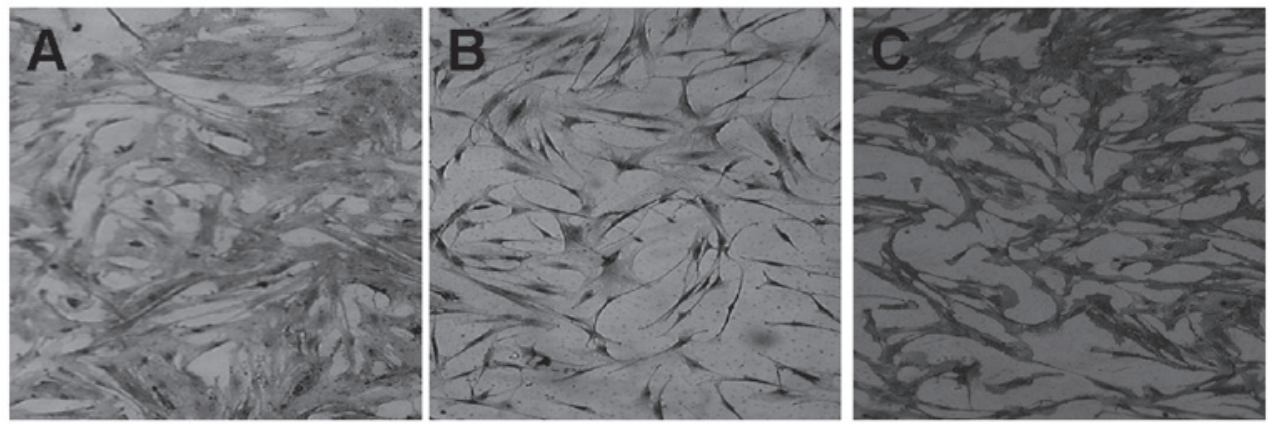

Figure 2. Staining of MSCs (magnification, x200). (A) Incubation of human bone marrow MSCs with CD105 primary antibody resulted in insoluble precipitate at the antigen site. (B) Incubation of MSC linewithout CD 105 primary antibody resulted in no insoluble precipitate at the antigen site (negative control) (C) Incubation of positive MSC line obtained from American Type Culture Collection (Rockville, MD, USA) was treated with CD 105 primary antibody resulted in an insoluble precipitate at the antigen site. MSC, mesenchymal stem cells; CD 105, cluster of differentiation 105.

Germany). Absorbance was read immediately at $405 \mathrm{~nm}$ with a reference wavelength of $630 \mathrm{~nm}$ on the ELISA Reader (Sunrise $^{\mathrm{TM}}$; Tecan Group Ltd., Mannedorf, Switzerland).

Matrix mineralization staining. Von Kossa staining was used to identify the matrix mineralization after three weeks in osteogenic induction culture. In brief, $1 \mathrm{ml} 5 \%$ silver nitrate was added to the culture plate and the cells were exposed to $100 \mathrm{~W}$ lamp light for $60 \mathrm{~min}$. The slide was rinsed and placed in $0.5 \%$ hydroquinone for $2 \mathrm{~min}$ and then washed with distilled water. A total of $1 \mathrm{ml} 5 \%$ sodium thiosulfate solution was added for $2 \mathrm{~min}$ and rinsed with distilled water. The slide was stained with Biebrich Scarlet-Acid Fuchsin and observed under the microscope for mineralized extracellular matrix.

Statistical analysis. All experimental analyses were performed in triplicate $(\mathrm{n}=3)$. Data are expressed as the mean \pm standard deviation. $\mathrm{P}<0.05$ was considered to indicate a statistically significant difference.

\section{Results and Discussion}

Cell adhesion and proliferation. MNC samples obtained from the human bone marrow were cultured and maintained in MSC growth medium. The morphology of the MSCs seeded for $24 \mathrm{~h}$ is shown in Fig. 1A. The results showed that the MSCs exhibited a fibroblast-like morphology and could attach well onto the plates. After 5 days, the adherent cells were composed of bipolar fibroblast-like cells that could later be grown to confluence in MSC growth medium (Fig. 1B and C). An attempt was also made to culture the MNCs in DMEM-10\% FBS medium; however, in contrast to the parallel cultures growing in MSC growth medium, cells growing in DMEM-10\% FBS did not expand or proliferate (Fig. 1D). Thus, MSC growth medium successfully supported the in vitro expansion of MSCs, as indicated by rapid proliferation and the appearance of large, oval-shaped, fibroblast-like cells.

Characterization of MSCs by histochemical staining. The use of bone marrow stem cells in gene and cell therapy requires in vitro expansion and culture conditions that preserve their differentiation and proliferative potential. MSC-like cells are fibroblastoid and express a marker protein typical of MSCs, CD 105. In this study, the cells were analyzed using histochemical staining with primary antibodies against CD 105, based on the labeled streptavidin-biotin method. The MSC surface marker reacted with the anti-CD 105 primary antibody, resulting in a fuchsia stain (Fig. 2). The results showed that CD 105 was positively expressed, and the purity of the third-passage MSCs was $>95 \%$. Subsequent to cell sorting and expansion, the pure and uniform MSCs were stocked as seeded cells for further study. The human bone marrow stem cells, which exhibit a multi-lineage capacity, were differentiated towards the osteogenic lineages using lineage-specific induction factors. In the present study, the induction of human bone 


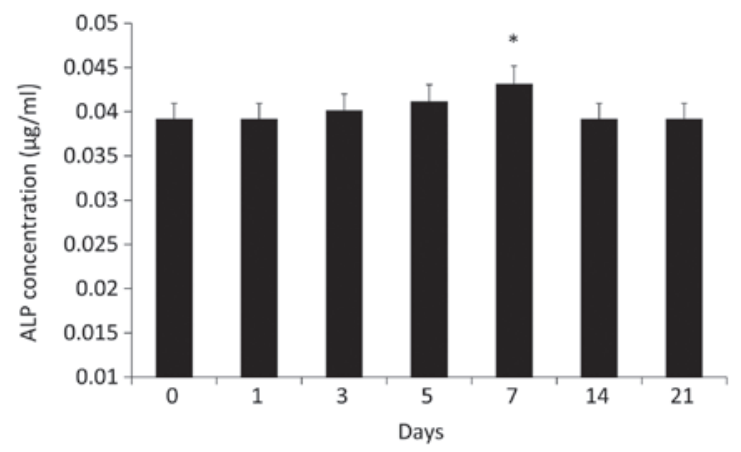

Figure 3. ALP activity of human mesenchymal stem cells. In a separate experiment, the cells were lysed after 0, 1, 3, 5, 7, 14 and 21 days in osteogenic media, and incubated with $p$-nitrophenol phosphate substrate to measure ALP activity. ALP, alkaline phosphatase. ${ }^{*} \mathrm{P}<0.05$ vs. 0 days.

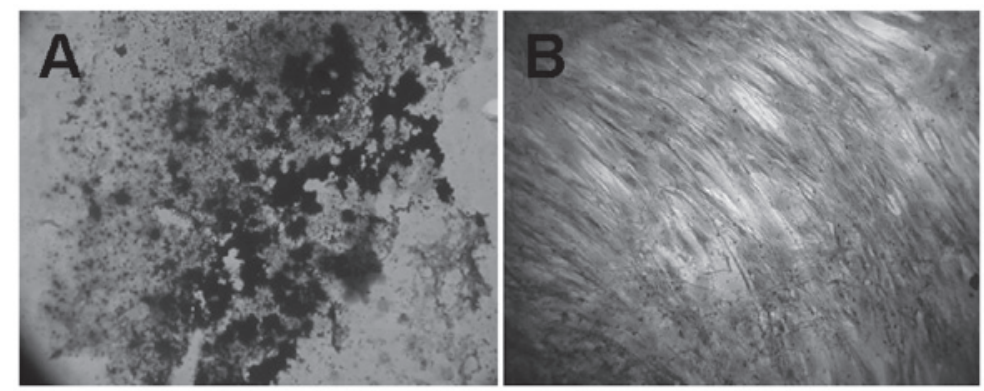

Figure 4. MSC-driven osteogenesis in monolayer cultures (magnification, x400). (A) Positive von Kossa staining in a monolayer culture of human bone marrow-derived MSCs exposed to osteogenic medium for three weeks. (B) Control monolayer cultures of MSCs grown with basic medium (no osteogenic supplement) for three weeks showed a negative reaction to von Kossa staining. MSC, mesenchymal stem cell.
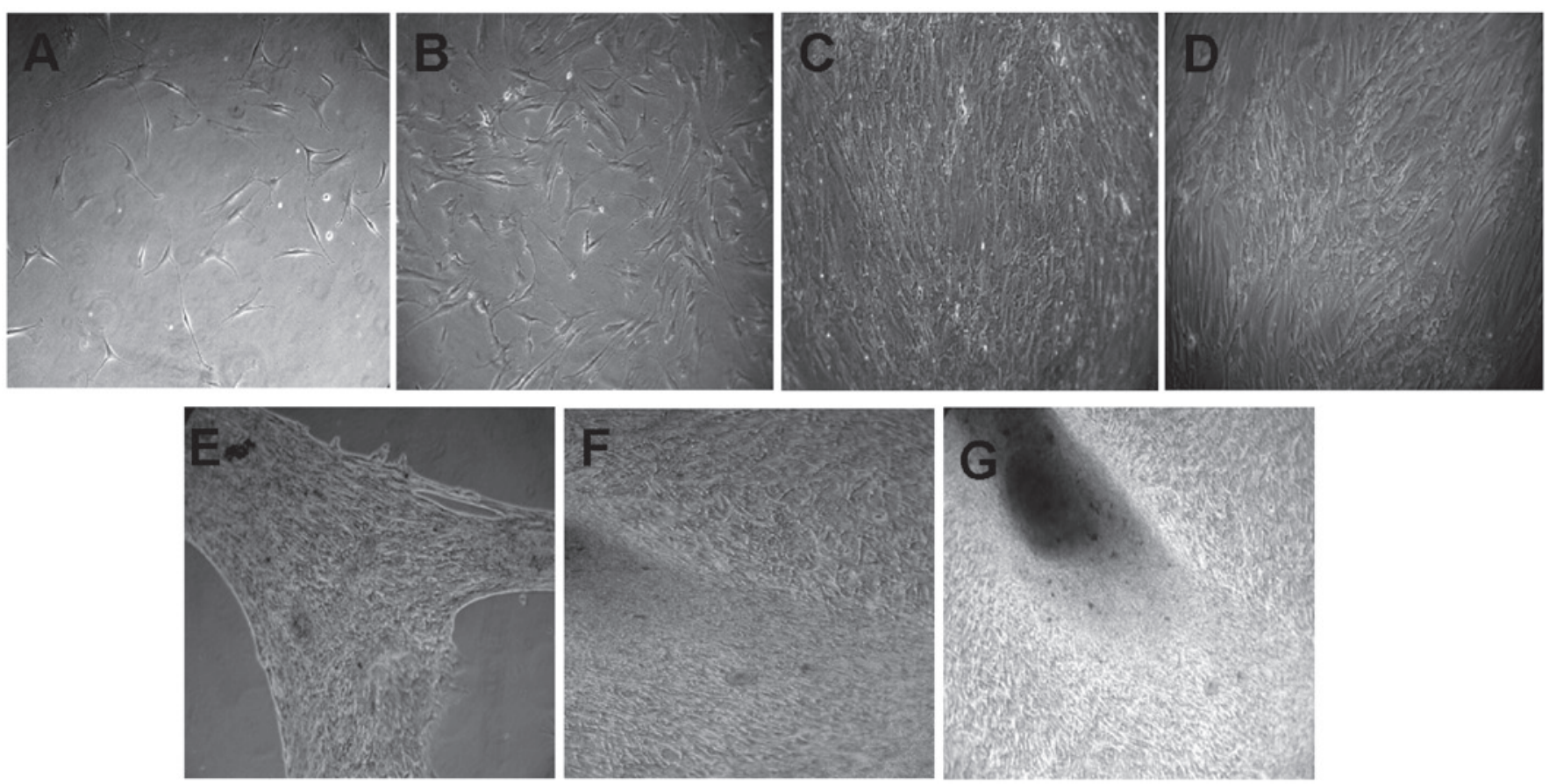

Figure 5. Morphology of mesenchymal stem cells in osteogenic media. (A) Day 0 (magnification, x100); (B) day 2 (magnification, x100); (C) day 5 (magnification, x200); (D) day 7 (magnification, x200); (E) day 14 (magnification, x200); (F) day 21 (magnification, x200); (G) day 28 (magnification, x200).

marrow MSCs in osteogenic media in the presence of ascorbic acid, $\beta$-glycerol phosphate and dexamethasone showed that the MSCs had a multi-lineage potential. Dexamethasone is a synthetic glucocorticoid shown to induce osteogenic differentiation in MSCs, and $\beta$-glycerol phosphate promotes mineralized matrix formation by acting as a potential source of phosphate ions (9). Ascorbic acid is a cofactor required for the function of several hydroxylases. It has been shown that 
ascorbic acid, together with $\beta$-glycerol phosphate and dexamethasone, induces embryonic stem cells to differentiate into mineralized osteoblasts in vitro (10).

Biochemical analysis of ALP. ALP activity is one of the most commonly used markers for osteogenesis, since it reflects the proportion of osteogenically differentiated cells. ALP activity was determined from a $p$-nitrophenol standard curve. Incubation of human MSCs under the osteogenic condition within one week resulted in a marked increase in ALP activity. As shown in Fig. 3, increased ALP activity was observed in human MSC cultures on day 2, with the increase peaking on day 7. However, the activity then decreased between days 7 and 14. This may have been a result of the cells reaching confluence earlier due to rapid proliferation, which may have resulted in earlier differentiation. Intercellular communication affects the expression of differentiation markers in osteoblasts (11); therefore, a higher cell density likely results in a higher degree of cell-cell interaction, and thus a higher rate of differentiation.

Von Kossa staining of osteogenic differentiated MSCs. To confirm osteogenic differentiation, calcification of the matrix was assessed in MSCs using the von Kossa Stain. Calcification appeared as black regions within the cell monolayer. Consistent with osteogenesis, several black regions, indicative of calcified extracellular matrix, red regions, indicative of the nucleus, and light pink regions, indicative of the cytoplasm, were observed in human bone marrow stem cells treated for three weeks in osteogenic medium (Fig. 4A). No calcification (black region) was observed in the undifferentiated MSCs (Fig. 4B), although red and light pink regions, indicative of the nucleus and cytoplasm, respectively, were observed. The three-week incubation period with osteogenic-inducing culture medium resulted in an accumulation of calcium deposits. Monolayer cultures of human bone marrow MSC-derived osteogenic cells showed a positive reaction to von Kossa staining, relative to the control monolayer MSC cultures that were incubated with regular culture medium without the osteoinductive supplement. Bone marrow has been reported to be the most established, best-understood and most reliable source of skeletal stem cells $(12,13)$. These cells have already shown great regenerative potential. During the 28 -day assay period, MSCs cultured with optimized osteogenic medium underwent a marked change in cellular morphology. The cultures began to form multilayered nodular structures, as the apparent result of coalescing cellular aggregates, and nearly all cells of the nodular structures were cuboidal, as shown in Fig. 5. The induction of human MSCs using osteogenic medium was examined. The results indicated that, in comparison with the MSC growth medium, MSCs cultured in medium containing osteogenic supplements formed cell clusters and mineral deposits. The majority of the cells that attached to the plastic surface exhibited a fibroblast-like spindle shape. The cells proliferated to form a uniform confluent cell monolayer and became cuboidal in shape five days after changing from the
MSC growth medium to the osteogenic medium (Fig. 5). The cells began to coalesce between days 14 and 28 . Overall, this study may provide some useful information on the effect of MSC growth medium and its stimulation of rapid proliferation and osteogenic differentiation.

Considerable evidence has definitively demonstrated that MSCs exist in adult tissues or organs. Although the collection of bone marrow is time consuming and painful, bone marrow has relatively high numbers of MSCs and has high osteoblast potential. The modified method utilized in the present study resulted in the rapid proliferation of MSCs. These findings suggest that this medium has promising potential for the further analysis of bone tissue engineering of MSCs. To continue to take advantage of these cells for cell and gene therapy applications, however, requires a complete understanding of MSC culture maintenance and how the differentiation of MSCs is regulated in vivo and in vitro. Knowledge gained in these areas is likely to facilitate the design of optimal in vitro conditions that incorporate regimes targeted towards generating highly functional MSCs for cell-based clinical application.

\section{References}

1. Price JS, Oyajobi BO and Russell RG: The cell biology of bone growth. Eur J Clin Nutr 48 Suppl 1: S131-S149, 1994.

2. Sanchez-Ramos J, Song S, Cardozo-Pelaez F, et al: Adult bone marrow stromal cells differentiate into neural cells in vitro. Exp Neurol 164: 247-256, 2000

3. Horwitz EM, Gordon PL, Koo WK, et al: Isolated allogeneic bone marrow-derived mesenchymal cells engraft and stimulate growth in children with osteogenesis imperfecta: Implications for cell therapy of bone. Proc Natl Acad Sci USA 99: 8932-8937, 2002.

4. Koç ON, Gerson SL, Cooper BW, et al: Rapid hematopoietic recovery after coinfusion of autologous-blood stem cells and culture-expanded marrow mesenchymal stem cells in advanced breast cancer patient receiving high-dose chemotherapy. J Clin Oncol 18: 307-316, 2000.

5. Petite H, Viateau V, Bensaïd W, et al: Tissue-engineered bone regeneration. Nat Biotechnol 18: 959-963, 2000.

6. Ohgushi H, Miyake J and Tateishi T: Mesenchymal stem cells and bioceramics: strategies to regenerate the skeleton. Novartis Found Symp 249: 118-127, 2003.

7. Hung SC, Chen NJ, Hsieh SL, et al: Isolation and characterization of size-sieved stem cells from human bone marrow. Stem Cells 20: 249-258, 2002.

8. Friedenstein AJ, Latzinik NW, Grosheva AG and Gorskaya UF: Marrow microenvironment transfer by heterotopic transplantation of freshly isolated and cultured cells in porous sponges. Exp Hematol 10: 217-227, 1982.

9. Shin H, Temenoff JS, Bowden GC, et al: Osteogenic differentiation of rat bone marrow stromal cells cultured on Arg-Gly-Asp modified hydrogels without dexamethasone and beta-glycerol phosphate. Biomaterials 26: 3645-3654, 2005.

10. Carinci F, Pezzetti F, Spina AM, et al: Effect of Vitamin C on pre-osteoblast gene expression. Arch Oral Biol 50: 481-496, 2005.

11. Li Z, Zhou Z, Yellowley CE and Donahue HJ: Inhibiting gap junctional intercellular communication alters expression of differentiation markers in osteoblastic cells. Bone 25: 661-666, 1999.

12. Minguell JJ, Erices A and Conget P: Mesenchymal stem cells. Exp Biol Med (Maywood) 226: 507-520, 2001.

13. Wexler SA, Donaldson C, Denning-Kendall P, et al: Adult bone marrow is a rich source of human mesenchymal 'stem' cells but umbilical cord blood and mobilized adult blood are not. Br J Haematol 121: 368-374, 2003. 